\title{
Afflux des migrants
}

\author{
Patrick Bodenmanna, Françoise Ninane ${ }^{\mathrm{b}}$, Brigitte Pahud-Vermeulena, Elodie Dory ${ }^{\mathrm{a}}$, Martine Monnat ${ }^{\mathrm{c}}$, \\ Jacques Cornuz ${ }^{\mathrm{b}}$, Eric Masserey ${ }^{\mathrm{c}}$ \\ ${ }^{a}$ Centre des populations vulnérables, Policlinique médicale universitaire, Lausanne; ${ }^{b}$ Policlinique médicale universitaire et \\ Département Universitaire de Médecine et Santé Communautaire, Lausanne; ${ }^{\circ}$ Service de Santé Publique du Canton de Vaud
}

Différents organismes internationaux (Eurostat, Organisation Internationale des Migrations, Frontex, Haut Commissariat des Réfugiés, National Geographic) estiment aujourd'hui qu'une personne sur 122 au niveau mondial est un déplacé interne, un requérant d'asile ou un réfugié statutaire, représentant la population du $24^{\mathrm{e}}$ pays le plus peuplé du monde! Le nombre de personnes déracinées par la guerre est le plus important depuis la Deuxième Guerre mondiale selon le HCR. D'autres chiffres et données nous permettent de mieux comprendre pourquoi ces mouvements migratoires sont considérés comme historiques aujourd'hui: 42500 personnes en moyenne se sont déplacées chaque jour en Europe en 2014, 3000 sont décédées dans la mer méditerranée en 2015, les principaux pays d'émigration étant la Syrie (1,9 millions ont trouvé refuge en Turquie et $25 \%$ de la population du Liban est syrienne), le Kosovo, l'Afghanistan, l'Albanie et l'Irak (données janvier-juin 2015). Le retentissement médiatique est énorme. Le 19 octobre 2015, la revue TIME consacre plus de la moitié de son numéro (52 pages sur 96 !) à la crise migratoire européenne intitulant ce numéro spécial Exodus. The epic migration to Europe and what lies ahead [1]*.

\section{Les migrants forcés actuels présentent} une «clinique de la pauvreté».

Ces chiffres démontrant une migration d'ampleur vraiment historique s'intègrent dans une histoire qui ne cesse de se répéter à plus ou moins grande échelle. Après la Première Guerre mondiale, entre 1917 et 1939, la Révolution russe et la chute de l'Empire ottoman provoquaient la fuite de 5 millions de personnes cherchant refuge et la création du passeport Nansen pour quelques 450000 apatrides. Plus de 11 millions de personnes ont été déplacées lors de la Seconde Guerre mondiale, et la Convention de Genève de 1951 a défini le statut de réfugié. En 1975, suite aux victoires de gouvernements communistes, trois millions de vietnamiens, laotiens et cambodgiens quittaient leur pays et les boatpeople faisaient leur apparition. Enfin, durant la fin des années 90, plusieurs dizaines de milliers de kosovars quittaient la guerre en ex-Yougoslavie en demandant l'asile politique notamment à la Suisse [2].

\section{Besoins sanitaires des migrants}

Selon le BMJ, les migrants forcés actuels présentent une «clinique de la pauvreté»: déshydratation, fatigue extrême, lésions aux pieds, infections cutanées, traumatismes mineurs et atteintes de la santé mentale dans un contexte de chaos; ainsi les médecins de famille, pédiatres, gynécologues et psychiatres seraient les acteurs nécessaires pour faire face à ces besoins sanitaires [3]. Par contre, le risque de maladies infectieuses importées est mineur selon l'Organisation Mondiale de la Santé (OMS) qui souligne plutôt le risque de soins inéquitables à ces populations vulnérables dans les pays de transit ou d'accueil [4]. Le HCR plaide pour la prise en charge des migrants forcés dans les systèmes sanitaires des pays d'accueil [5]. Concernant spécifiquement les réfugiés syriens, "the biggest refugee population from a single conflict in a generation", le HCR souligne qu'au Liban où actuellement $1 / 4$ de la population est constituée de personnes fuyant le régime syrien, les principaux enjeux sanitaires sont les femmes enceintes et les quelques 142000 enfants nés dans l'exode depuis 2011, ainsi que la gestion des maladies chroniques telles que le diabète et autres risques cardiovasculaires [6, 7].

Des éditoriaux du Lancet nous rappellent d'abord que la Conférence d'Evian de 1939 dans le cadre du début de la Seconde Guerre mondiale avait déjà réduit les processus migratoires des persécutés de l'époque à des nombres, mettant sous silence notamment la santé de ces personnes et en particulier la santé des enfants [8]. De même, il est fait mention du rôle potentiel des diasporas au service de ces réfugiés dans les pays hôtes et en particulier de la diaspora syrienne face aux événements actuels, tels que cela a été le cas lors des récentes tragédies au Népal, aux Philippines, en Chine, à Haïti ou avec l'épidémie d'Ebola [9].

La première enquête sanitaire des migrations forcées de ces derniers mois est parue dans le NEJM récemment [10]: elle fait mention de l'arrivée de plus de 410000 migrants dont $25 \%$ au mois d'août et de 20000 personnes en un seul week-end à Munich. L'adaptation sanitaire a nécessité la mise en place de tentes autour de la gare de Munich, avec un soutien interdisciplinaire d'équipes de médecins, infirmiers, psychologues 
et psychiatres dont un grand nombre a travaillé de manière bénévole; la prise en charge s'est faite en deux temps avec initialement un tri rapide pour dans un deuxième temps, une intervention plus soutenue dans des structures plus adaptées.

Ces différentes informations nous confortent dans l'idée de besoins sanitaires spécifiques nécessitant une réponse "généraliste» interdisciplinaire qui doit être rapide, adaptée aux besoins des nouveaux réfugiés, efficace et la plus efficiente possible.

\section{Le dispositif vaudois: une réponse}

Le canton de Vaud accueille 8,4\% des requérants d'asile qui demandent l'asile politique à la Suisse. Pour répondre aux demandes sanitaires de cette population, le Canton a créé en 1998 un réseau - aujourd'hui appelé Réseau de Santé et Migration (RESAMI) - qui travaille conjointement avec les structures d'hébergement de l'Etablissement Vaudois d'Accueil des Migrants (EVAM). Ce réseau est constitué de 170 médecins de famille et pédiatres répartis sur l'ensemble du canton et de l'Unité de Soins aux Migrants (USMi), pilier et première ligne du réseau, formé de 12.6 Equivalent Plein Temps (EPT) infirmiers de première ligne, 9.1 EPT collaborateurs administratifs en soutien et 0.4 EPT de supervision médicale. Sur mandat du Service de la Santé Publique (SSP) du Canton de Vaud, les missions de l'USMi consistent en un bilan de santé pour chaque bénéficiaire (soit quelques 6000 requérants d'asile actuellement), un programme de vaccination pour l'ensemble des requérants d'asile, de la prévention/promotion de la santé ainsi que des consultations de pre-

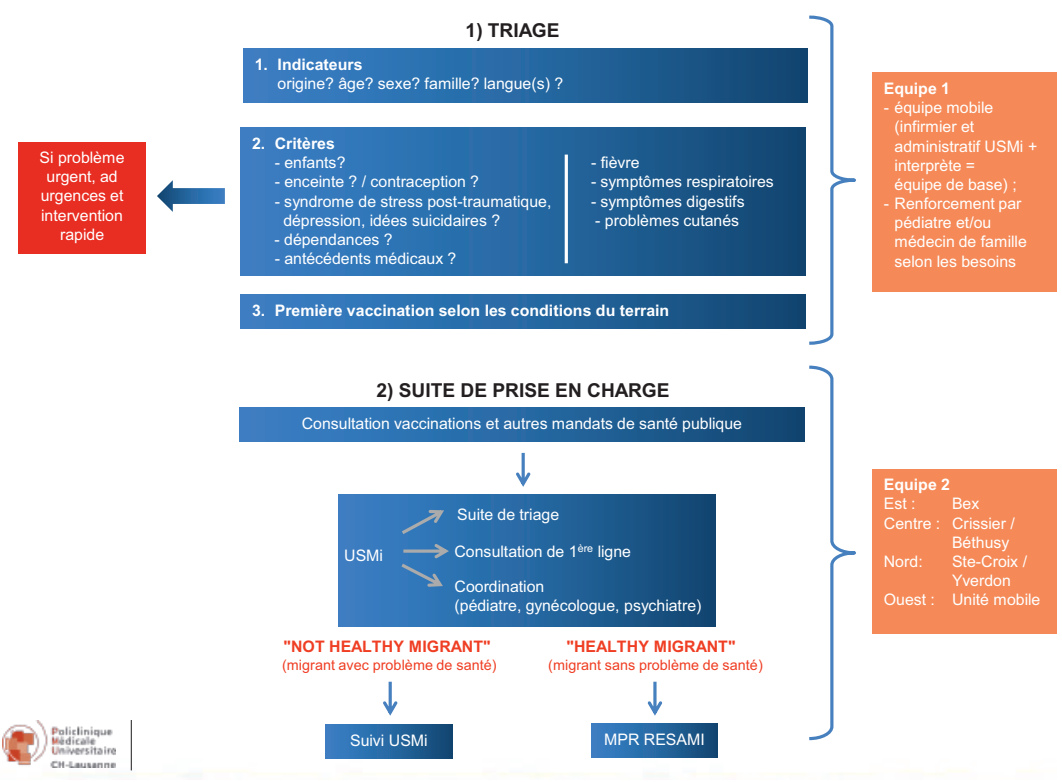

Figure 1: Dispositif vaudois de crise face à l'afflux des migrants.
Groupe de travail ayant participé à l'élaboration du dispositif de crise.
L. Bouche (PMU)
L. Wenger (PMU),
Y. L'Hostis (PMU)
B. Perrault (PMU)
C. Marteau (PMU)
A. Rosat (PMU)
G. Seroux (PMU)
M. Gehri (HEL-CHUV)
F. Maillefer (PMU)
S.-C. Renteria (DGOG-CHUV)
R. Marion-Veyron (PMU)
S. Depallens-Villanueva
(DISA-CHUV)
M. Grand (PMU)
P. Rochat (EVAM)

PMU: Policlinique médicale universitaire

HEL-CHUV: Hôpital de l'Enfance, Centre hospitalier universitaire vaudois DGOG-CHUV: Département de Gynécologie et Obstétrique DISA-CHUV: Division interdisciplinaire de santé des adolescents EVAM: Etablissement Vaudois d'Accueil des Migrants

mière ligne sous le format de gate-keeping infirmier; sur les plus de 30000 actes annuels effectués au cours des 3 dernières années, $70 \%$ des patients n'ont été vus que par l'USMi, seuls 30\% d'entre eux nécessitant une prise en charge médicale ou institutionnelle [11]. Audelà de la consultation de première ligne, l'USMi poursuit sa prise en charge et son orientation pour une partie des patients les plus vulnérables. Cette équipe interdisciplinaire intervient sur 7 sites distincts du canton de Vaud (Lausanne, Ste-Croix, Yverdon, Nyon, Bex, Crissier, Vevey) et le réseau a été évalué à plusieurs reprises: la qualité des soins prodigués [12], le vécu des soignants et des soignés [13] et son efficience [14] donnent satisfaction.

Cette équipe a été conçue pour prendre en charge 80 à 100 nouvelles arrivées par mois. Depuis 5 mois, les nouvelles arrivées sont supérieures à 300 personnes par mois (385 en septembre) [15]. Afin de faire face à la crise migratoire et l'afflux massif de réfugiés des derniers mois, un fonctionnement en «mode urgence» a été établi en redimensionnant l'ensemble des activités de l'USMi, avec notamment le temps alloué aux bilans de santé, et en suspendant toutes les activités spécifiques de prévention et promotion de la santé. Nous avons également fait appel à d'autres services de la PMU pour venir en renfort de l'équipe USMi. Cet effort d'adaptation à dispositif et personnel égaux s'est cependant avéré insuffisant face à l'importance des besoins. Après un travail de définition des prestations et d'organisation, travail regroupant praticiens et responsables de l'USMi, une augmentation d'un tiers des ressources pour répondre à un afflux trois à quatre fois supérieur a été proposée. Ce développement des capacités de l'USMi a fait l'objet d'un aval à la fois institutionnel et politique.

Sur base de l'analyse de la littérature récente, et en concordance avec les autorités sanitaires du canton, nous avons mis sur pied un dispositif de crise pour répondre à l'évolution des besoins (fig. 1): 


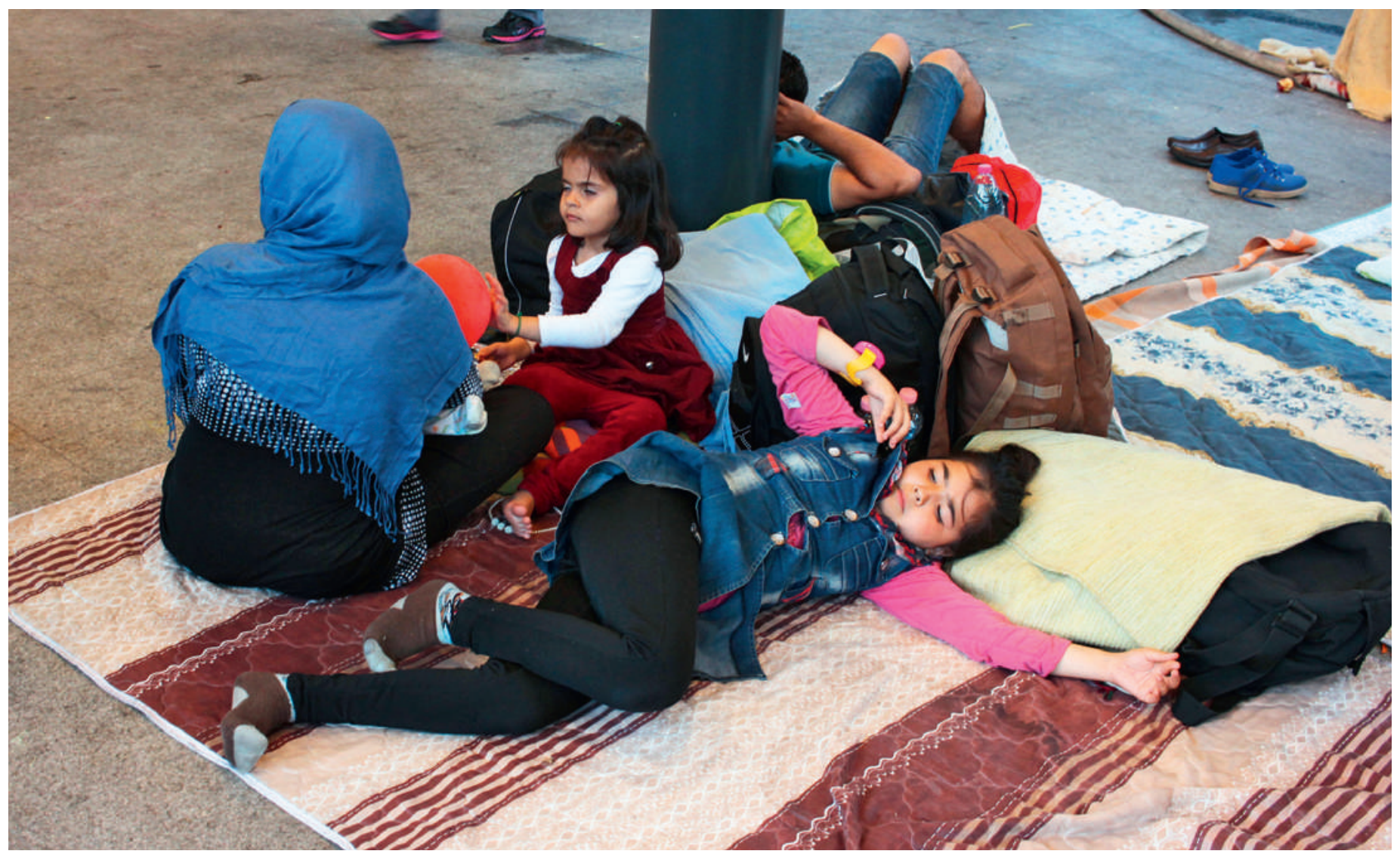

Les réfugiés ont des besoins sanitaires spécifiques nécessitant une réponse "généraliste» interdisciplinaire.

- L'équipe mobile (équipe 1) a pour tâche d'effectuer des consultations courtes sous forme de triage, orientation en cas de besoin et premières vaccinations sur les lieux d'hébergement EVAM pour tous les nouveaux arrivés. Cette équipe est constituée de deux infirmiers et d'un collaborateur administratif, si nécessaire d'un pédiatre et/ou d'un médecin de premier recours. L'approche se base sur des indicateurs et critères anamnestiques simples et une approche syndromique ciblant certaines pathologies: maladies pulmonaires infectieuses, atteintes du tractus digestif, problèmes dermatologiques. Au besoin, l'anamnèse est complétée par un bilan ciblé. Le but est d'identifier le plus rapidement possible les patients les plus fragiles pour garantir un maillage serré de sécurité sanitaire, et de les orienter vers les structures les plus adaptées à leurs besoins.

- L'équipe fixe (équipe 2), assure le suivi des vaccinations, une consultation de suite de triage pour tous les bénéficiaires, la coordination avec les différents partenaires ainsi que des consultations de première ligne pour les patients les plus vulnérables. Les patients dont la situation médicale le permet sont dirigés vers les médecins de famille et pédiatres du RESAMI pour la suite de leur prise en charge (groupe
Healthy migrant). Pour les situations plus complexes (groupe Not healthy migrant), il leur est proposé de poursuivre leur prise en charge au sein de l'USMi. Une veille sanitaire sur la base d'indicateurs informatisés permet de mieux cibler les interventions de l'USMi en fonction des besoins des réfugiés mais aussi de l'évolution des migrations.

Notre réponse locale se base sur l'expertise et l'effort de guidance de différents groupes de l'OMS au travers d'enquêtes sur la réponse à la crise des réfugiés de pays européens [16-18]. Cette démarche nécessite un besoin et une volonté des professionnels du terrain relayés aux décideurs (bottom-up) mais aussi l'acceptation et la volonté de ces derniers (top-down). Elle s'inscrit plus largement dans un courant de défense d'une cause prioritaire, d'advocacy tel que plébiscité récemment par l'un de nos confrères dans la Revue Médicale Suisse [19]: «Continuons chers et chères Collègues, à nous indigner, à prendre la défense de nos patientes et de nos patients en situation de vulnérabilité ou de dépendance, chaque fois que nous le jugeons nécessaire.»

Crédit photo

() Vasile $87 \mid$ Dreamstime.com 


\section{Références}

1 Vick K. The migrant crisis is a major test for European identity and unity. Time magazine. 2015:5-6.

2 Vick K. The Great Migration Time magazine. 2015:28-79.

3 Gulland A. The refugee crisis: what care is needed and how can doctors help? Bmj. 2015;351.

4 Gulland A. Refugees pose little health risk, says WHO. Bmj. 2015;351.

5 Abbasi K, Patel K, Godlee F. Europe's refugee crisis: an urgent call for moral leadership. Bmj. 2015;351.

6 Gornall J. Healthcare for Syrian refugees. Bmj. 2015;351

7 Ben Taleb Z, Bahelah R, Fouad FM, et al. Syria: health in a country undergoing tragic transition. International journal of public health. 2015;60 Suppl 1:S63-72.

8 Adapting to migration as a planetary force. Lancet. 2015;386(9998):1013.

9 Nagarajan N, Smart B, Nwadiuko J. Diaspora engagement in humanitarian emergencies and beyond. Lancet. 2015;386(9998):1015-6.

10 Nicolai T, Fuchs O, von Mutius E. Caring for the Wave of Refugees in Munich. The New England journal of medicine 2015;373(17):1593-5.

11 Puig F, Ninane F, Bodenmann P. [A new role: the gate-keeping nurse]. Krankenpflege Soins infirmiers. 2009;102(10):48-51, 70-1.
12 Bodenmann P, Althaus F, Burnand B, et al. Medical care of asylum seekers: a descriptive study of the appropriateness of nurse practitioners' care compared to traditional physician-based care in a gatekeeping system. BMC public health. 2007;7:310.

13 Ilario R, Marion D-M, Geneviève $\mathrm{P}$, et al. Professionnels infirmiers et usagers requérants d'asile dans un réseau de santé. Approche socio-anthropologique des politiques de santé. 2004.

14 Jean-Claude R, Laure C. Etude de solutions alternatives pour l'assurance des requérants d'asile 2004.

15 EVAM. Demandes asile suisse, nouveaux séjours, VD. Secondary Demandes asile suisse, nouveaux séjours, VD 2015. www.evam.ch/ fileadmin/groups/1/documents_pdf/Stat/150801_demandes asile suisse nouveaux sejours VD.pdf

16 Toolkit for assessing health-system capacity for crisis management WHO; 2012:39.

17 Second assessment of migrant health needs Lampedusa and Linosa, Italy. WHO 2012:34.

18 Expert opinion on the public health needs of irregular migrants, refugees or asylum seekers across the EU's southern and south eastern borders. 2015.

19 François P. Advocacy. Revue medicale suisse. 2015;486:1723. 